\title{
Trophectoderm and embryoblast proliferate at slow pace in the course of embryonic diapause in the roe deer (Capreolus capreolus)
}

\author{
AB Rüegg, S Bernal, FN Moser, I Rutzen and SE Ulbrich \\ Institute of Agricultural Sciences, ETH Zurich, Switzerland
}

Corresponding author e-mail: seu@ethz.ch

\begin{abstract}
The European roe deer (Capreolus capreolus) was the first mammal in which embryonic diapause has been described. While diapause is characterized by a complete developmental arrest in some species, roe deer blastocysts show a very slow, yet continuous growth. To date, it is neither known whether this growth is accompanied by developmental progression nor whether it is uniform in both, the trophectoderm (TE) and the inner cell mass (ICM). We collected roe deer blastocysts during the regular hunting season from September 2018 to January 2019, and quantified the fraction of cells expressing the proliferation marker Ki67 by immunofluorescence and light-sheet microscopy. We found that the cell number increased from around 300 cells in September to over $20^{\prime} 000$ cells per blastocyst in December before elongation occured. Concurrently, we observed considerable morphological changes, i.e. cavity formation and transition to a disk-like shape of the inner cell mass. During diapause, less than $10 \%$ of all cells displayed positive Ki67 staining. Strikingly, the relative increase in cell number was lower in the ICM compared to the TE, whereas the fraction of Ki67 positive cells appeared to be lower in the TE than in the ICM. Our findings thus confirm that roe deer blastocysts display developmental progression in the course of diapause. We hypothesize that while the overall duration of the cell cycle is longer in the ICM than in the TE, the fractional distribution of cell cycle phases differs, with TE cells having a longer $\mathrm{G} 1$ phase than cells of the ICM.
\end{abstract}

Keywords: Diapause, Embryo, Development, Proliferation, Differentiation

\section{Introduction}

In mammals, embryonic diapause describes the temporary arrest or deceleration of embryonic development at the blastocyst stage. Diapause allows the uncoupling of the time of mating from birth and thus allows progenies to be born when conditions are most favorable. To date, over 130 mammalian species, distributed across many taxa, are known to exhibit diapause [1]. Interestingly, even within mammals, diapause comprises a very heterogeneous phenomenon. Depending on the species, diapause can occur in every gestation (seasonal or obligate diapause), or can be induced by lactation (lactational or facultative diapause) [1]. The duration of diapause can vary from a few days up to several months. Furthermore, in some species, like mice, rats and kangaroos, blastocyst growth ceases completely, while in others, e.g. mustelids, bears, seals, and roe deer, limited, but continuous growth can be observed [1]. Despite first descriptions of diapause dating back to the $19^{\text {th }}$ century [2], the underlying mechanisms are still only poorly understood. The thorough 
characterization of the phenotypical differences is inevitable to understand how the molecular mechanism of diapause induction and reactivation are regulated in different species.

The European roe deer (Capreolus capreolus) was the first species for which diapause has been described [2]. However, our understanding of the cellular processes in the blastocyst occurring in the course of diapause is still very limited. The roe deer is a monoestrous seasonal breeder [3]. The rut and subsequent ovulation take place from late July to early August [4-6]. On average, two corpora lutea $(\mathrm{CL})$ are formed and maintained for five months regardless of whether or not a pregnancy is established [4]. The CL continuously secrete progesterone throughout pregnancy [7]. After fertilization, the cleaving embryo passes through the oviduct and a blastocyst, composed of inner cell mass (ICM) and trophectoderm (TE), is formed. The blastocyst hatches from the zona pellucida before the end of August. After hatching, the developing blastocyst remains unattached in the uterus until the end of December/beginning of January. In this period, the blastocyst grows from about 0.1 to $3 \mathrm{~mm}$ in diameter. Whether this growth is accomplished by cellular proliferation, cell growth, or a combination of both is still unclear. Upon reactivation about 4-5 months post fertilization, the blastocyst rapidly elongates until it spans the entire length of the uterine horn (10$15 \mathrm{~cm}$ ) and implantation takes place. The developmental period is thus immensely slower compared to other ruminant species, i.e. in cattle elongation starts around day 14 post estrus [810].

To our knowledge, the embryonic developmental progression, i.e. the differentiation of cells into distinct embryonic cell lineages, in the course of roe deer diapause has only been described in a single histological study by Keibel in 1902 [11]. Keibel's micrographic drawings clearly point toward the latter. Multiple drawings clearly display hypoblast formation, cavitation and the formation of an embryonic disc in the ICM during diapause. While we are aware of these potential changes, we will generally refer to the embryonic compartment in roe deer as ICM. Based on histological sections of individual roe deer blastocysts, it has been described that during diapause the blastocysts have a rather low mitotic index [4, 11]. Lengwinat and Meyer performed a BrdU incorporation assay [12] and revealed that in October and November, less than $5 \%$ of trophoblast cells underwent DNA synthesis within a $4 \mathrm{~h}$ culture period. In late November and December, the percentage of BrdU positive cells increased to over $10 \%$ [12]. Yet, BrdU incorporation was not observed at any stage in the ICM. This contradicts the results earlier obtained by Keibel [11], who observed mitotic cells in both, ICM and trophoblast. Lengwinat and Meyer concluded that the absence of DNA synthesis in the ICM could have been imposed due to either the conditions or the duration of the blastocyst culture. Alternatively, the authors proposed that the growth rate of the ICM cells could have been lower than that of the TE cells [12].

While there are a variety of possibilities to assess cell proliferation, many of them require the use of transgenic cells or animals expressing reporters like FUCCI or culture in the presence of nucleotide analogues (e.g. BrdU, EdU). As the former is currently unfeasible and the latter can not rule out in vitro artefacts, immunofluorescence staining for cell cycle markers is the most feasible approach to be applied in roe deer. Ki67 is a widely used marker for detecting actively proliferating cells and serves as a prognostic marker for grading various types of cancer [13, 14]. Ki67 is expressed during the $\mathrm{S}$ to $\mathrm{M}$ phases of the cell cycle and is rapidly degraded after mitosis. Quiescent cells residing in the G0 phase of the cell cycle show very little to no Ki67 expression [15]. As such, an anti-human Ki67 antibody has already been used to characterize proliferation in roe deer testes and fetuses $[16,17]$. To investigate the proportion of proliferating cells in roe deer blastocysts during diapause and to assess the proliferation rate in both the ICM and the TE cells, we analyzed the fraction of cells expressing Ki67 in whole mount roe deer blastocysts using fluorescence immunohistochemistry and light sheet microscopy.

\section{Materials and methods}




\section{Blastocyst collection}

Uteri of 113 roe deer does were collected during regular huntings from September 2018 to January 2019 in Switzerland and southern Germany. The sample collection did not require ethical approval, since it was performed during regular huntings and none of the animals were culled for scientific purposes. The uteri were flushed using $2.5 \mathrm{ml}$ phosphate buffered saline (PBS) [Thermo Fisher Scientific, Waltham, USA] and in total 200 blastocysts were recovered. The blastocysts were imaged using a Zeiss SteREO Discovery.V8 stereomicroscope equipped with an Olympus SC-50 camera. The diameters of blastocysts and, if possible, the ICM were determined using the Olympus cellSens Standard software. Blastocysts were washed three times in PBS + 0.1\% poly-vinyl-alcohol (PBS-PVA) [Sigma-Aldrich, St. Louis, USA], fixed for $30 \mathrm{~min}$ in $3.7 \%$ paraformaldehyde (PFA) [Sigma] in PBS, washed again three times for $10 \mathrm{~min}$ in PBS-PVA and stored on ice or at $4^{\circ} \mathrm{C}$ in PBS-PVA $+1 \%$ Penicillin-Streptomycin [Thermo]. From the 200 blastocysts collected, we selected 57 for Ki67 localization. With respect to the sampling date, we selected a maximum number of blastocysts for histological processing with which we obtained an equal distribution for all sampling dates (Figure $1 \mathrm{E})$. As we were unable to find a suitable antibody to specifically stain either ICM or TE cells in roe deer, we assessed the localization of the ICM based on the morphological characteristics. Therefore, we selected blastocysts that had remained spherical or which were only moderately folded, at best not collapsed. As a consequence, the average of the blastocyst diameter in the stained blastocysts was slightly higher compared to all collected blastocysts (Figure 1D). Nevertheless, since folding most likely has been caused by the sampling procedure, we assumed that the selected population is representative of the physiological status.

\section{Blastocyst staining}

Single blastocysts were washed three times for 10 min in PBS-PVA + $0.1 \%$ Tween20 [Sigma] (PBSTP) and permeablized for $30 \mathrm{~min}$ in $0.5 \%$ TritonX-100 [Sigma], $10 \mathrm{mM}$ sodium citrate [Sigma] and $0.1 \%$ PVA in PBS and washed again three times for 10 min in PBSTP. Any unspecific binding sites were blocked by incubation in $2.5 \%$ BSA [Sigma] in PBSTP as blocking buffer for $2 \mathrm{~h}$. The Ki67 antibody (Monoclonal Mouse Anti-Human Ki-67, M724029-2 [Agilent, Santa Clara, USA]) has been previously described in histological examinations of roe deer embryos [16] and shows cross reactivity in a broad variety of species including sheep [18, 19], dog [20, 21] and pig [22]. The antibody was diluted $1: 100$ in blocking buffer and incubated over night at $4^{\circ} \mathrm{C}$. The next day, the blastocysts were washed three times for 10 min each in PBSTP and incubated with the secondary antibody (Goat Anti-Mouse IgG H\&L (Alexa Fluor ${ }^{\circledR}$ 594), ab150116 [Abcam, Cambridge, UK]), and washed again three times in PBSTP. The nuclei were stained by incubating in $0.2 \mathrm{ug} / \mathrm{ml} 4^{\prime}, 6-$ Diamidin-2-phenylindol (DAPI) [Sigma] in PBSTP for 10 minutes. After nuclear staining, the blastocysts were transferred to PBSTP and mounted in $1.2 \%$ low melting point agarose [Sigma] at $42^{\circ} \mathrm{C}$.

\section{Lightsheet microscopy}

All blastocysts undergoing Ki67 staining were imaged at the center for microscopy of the University of Zurich (ZMB) using a Zeiss Lightsheet - Zeiss Z.1 microscope equipped with $405 \mathrm{~nm}(20 \mathrm{~mW})$ and $561 \mathrm{~nm}(50 \mathrm{~mW})$ solid state diode lasers. The filtersets dapi-draq5 (Ip 490, bp 420-470, Ip 660) and gfp-mcherry (Ip 560, bp 505-530, Ip 585) were used. The 5x EC Plan-NEOFLUAR as well as 10x and 20x W Plan APO objectives were used and images were captured using two 3.8 MPixel sCMOsCameras. Image acquisition and microscope control was done using the ZEN Black Light sheet software. All pictures were acquired in 16 bit format. 


\section{Image analysis}

Images were normalized in FIJI/ImageJ (version 1.51p (23)) and objects were detected using the Imaris software (Version 9.3.0, Oxford Instruments). We used the nuclear DAPI staining to assess the number of cells for each blastocyst. The nuclei of the TE were detected using the Spots function with the following parameters: diameter of largest sphere for local contrast of $10 \mu \mathrm{m}$, spot quality above 1000. Ki67 was detected as a surface object to avoid recognizing small particles occasionally appearing outside of nuclei. The parameters were the following: smoothing of surfaces by $1 \mu \mathrm{m}$, background subtraction with minimal threshold of $10^{\prime} 000$ and diameter of largest sphere for local contrast of $16 \mu \mathrm{m}$, seed point diameter for watershed of $7 \mu \mathrm{m}$, quality of seed points above 1000, volume above $500 \mu^{3}$. While automatic detection of nuclei worked well for TE cells, the cells of the ICM were too dense to obtain an accurate cell count. Therefore, for both DAPI and Ki67 staining, positively stained cells of the ICM were manually annotated. To estimate the number of nuclei within the ICM, the images obtained from the ICM region were deconvolved using the Huygens Professional software (Version 19.10, Scientific Volume Imaging B.V.). For deconvolution, the following parameters were used; threshold for background subtraction: 2000, maximum number of iterations: 40 , SNR: 20 , quality threshold: 0.01 . For six blastocysts, the surface objects were created in Imaris as a first estimation, which was then corrected by manual counting. The ICM volume was detected using the following parameters for surface object creation: smoothing of surfaces by $2 \mu \mathrm{m}$, background subtraction with minimal threshold of 1000 and diameter of largest sphere for local contrast of $200 \mu \mathrm{m}$, volume threshold of 10'000 $\mu^{3}$. These ICM objects created by the software were manually curated and any falsely created objects were manually removed. Subsequently, the number of nuclei in the ICM was extrapolated based on the ICM volume.

\section{Data analysis}

The statistical analysis and the plotting of the data was performed in R (Version 3.6.1 (24)) using built-in functions and the tidyverse package collection. Unless stated otherwise, data are presented as means \pm SEM. The Shapiro-Wilk test was used to assess the normality distribution of both raw as well as log-transformed data. To estimate and quantify linear correlations among continuous variables, we used Pearson's correlations and/or Students t-tests. To quantify differences among non-parametric/categorical variables, we used Spearman's correlations and/or Mann-Whitney U tests. Regression models were created using the Im function. Residual, Normal Q-Q and Cook's distance plots were assessed for each model generated using the autoplot function of ggfortify. If the regression model was generated using log-transformed data, the coefficients of the regression line equations were transformed back to the linear scale for easier interpretation. The doubling time was calculated as $\ln (2) / k$, where $k$ is the slope of the linear regression line for the logtransformed number of nuclei against time (in days from first blastocyst collected). All plots were generated in the ggplot 2 package [25].

\section{Results and discussion}

Between September and January, the diameter of the blastocysts increased from approximately 0.3 $\mathrm{mm}$ to $3.5 \mathrm{~mm}$ (Figure $1 \mathrm{~B}$ ). In the same period, the diameter of the ICM increased from approximately $50 \mu \mathrm{m}$ to $350 \mu \mathrm{m}$ (Figure $1 \mathrm{C}$ ). Thus, in line with earlier studies $[4,11,26]$, we found a clear increase in blastocyst and ICM size in the course of diapause (Figure $1 \mathrm{~B}-\mathrm{C}$ ). Although we are aware that the relationship might not be linear, a linear correlation explained the data quite well (Pearson's $\mathrm{R}=0.73, \mathrm{t}=15.13, \mathrm{df}=199, \mathrm{p}$-value $<0.001$ and Pearson's $\mathrm{R}=0.66, \mathrm{t}=8.86, \mathrm{df}=101, \mathrm{p}$ value $<0.001$, for blastocyst and ICM size, respectively). On average, the ICM diameter was $8.5 \pm$ 
$2.5 \%$ of the blastocyst diameter. Interestingly, we noted that the ICM diameter did not increase at the same rate as the blastocyst diameter, with blastocysts smaller than about $2^{`} 000 \mu \mathrm{m}$ having a lower ICM increase than larger blastocysts (Figure 1F).

Using the blastocyst diameter to determine growth allows to analyze a large population of blastocysts relatively quickly. However, the extent to which the ICM and TE diameters can be compared is rather limited. The TE cells are distributed mainly across the surface of the blastocyst, while cells in the ICM are highly compacted. In addition, blastocysts have a tendency to fold and/or collapse to a variable degree during the sampling procedure [11]. Due to the compacted nature of the ICM, the folding of the blastocyst exerted a higher impact on the blastocyst diameter than on the ICM diameter. Thus, blastocyst folding might have lead to an overestimation of the relative growth of the ICM. In addition to the diameter, we therefore used the number of nuclei and the expression of a proliferation marker as additional growth measures.

The total number of nuclei in the blastocyst ranged from 313 to $25^{\prime} 997$ (Figure 2). We observed a positive log-log correlation (Pearson's $R=0.93, t=18.34, d f=55, p$-value $<0.001$ ) of the total number of cells with increasing blastocyst diameter (Error! Reference source not found.). From the generated linear regression model, we derived that a $100 \%$ increase in blastocyst diameter would lead to a $248 \%$ increase in the number of nuclei. By considering the blastocyst as a spherical object, the surface area increases with the squared diameter, namely displaying an exponential coefficient of 2 . The exponential coefficient of the correlation function was 1.8 and was not significantly different from 2 (Students $t$-test, $t=3.33, d f=56, p=0.27$ ). We thus conclude that the diameter may be used to approximate the cell number.

The number of nuclei counted in the course of diapause in the TE and ICM ranged from 284 to $24{ }^{`} 648$ and from 18 to $1^{`} 349$, respectively (Figure $3 A$ ). The fraction of cells in the ICM in relation to the total number of cells ranged from $2.0 \%$ to $13.0 \%$ with an average of $5.4 \pm 0.3 \%$. There was a weak negative correlation of the fraction of ICM cells with respect to the sampling date (Pearson's $R=-0.44, t=-3.66, d f=55, p$-value $<0.001)$. The log-transformed number of nuclei in the ICM was highly correlated with the log-transformed number of nuclei in the TE (Pearson's $R=0.92, t=17.43$, $\mathrm{df}=55, \mathrm{p}$-value $<0.001$ ). By applying a linear regression model (Adjusted $R 2=0.84$, F-statistic: $303.9, \mathrm{df}=55, \mathrm{p}$-value: $<0.001)$, the slope of the regression line was $0.84 \pm 0.05$ and significantly different from 1 (Students t-test, $t=3.33, d f=56, p<0.01$ ). We thus derived that a $79 \%$ increase in number of ICM nuclei would account for a $100 \%$ increase in number of TE nuclei. This indicates that overall, the relative increase in ICM nuclei was lower than the increase in TE nuclei (Figure 3A).

Nevertheless, a constant rate of increase in the context of diapause might not be adequate. Indeed, the ICM and the blastocyst diameter (Figure 1F) did not linearly increase in size in the course of embryo development. The slope of the LOESS regression appeared to change for blastocysts with more than about 3000 TE nuclei (Figure 3A) or with an blastocyst diameter of approximately 1500 $\mu \mathrm{m}$ (Figure 1F). Thus, the rate of increase in ICM nuclei relative to the TE nuclei may not be considered constant, with the ICM nuclei of smaller blastocysts increasing at a lower rate than ICM of larger blastocysts. However, in both cases the relative increase in ICM nuclei was lower than in TE nuclei. By comparing the number of ICM nuclei to the TE nuclei in the course of the sampling period (Figure $3 \mathrm{~B}$ ), we found that the relative increase of nuclei from mid-October until midDecember was lower in the ICM compared to in the TE. 
A

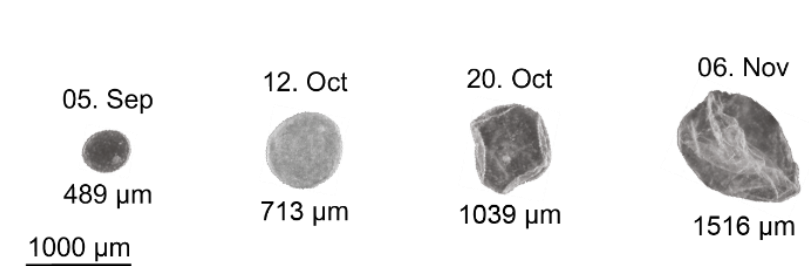

13. Dec

20. Nov

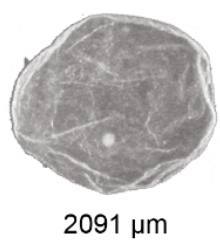

C

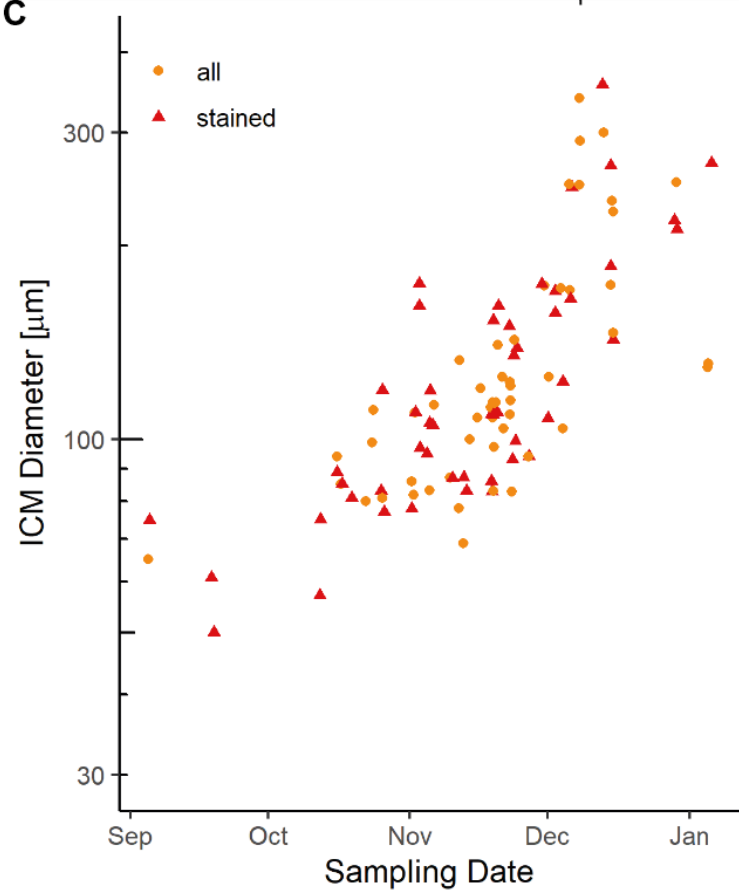

$\mathbf{F}$

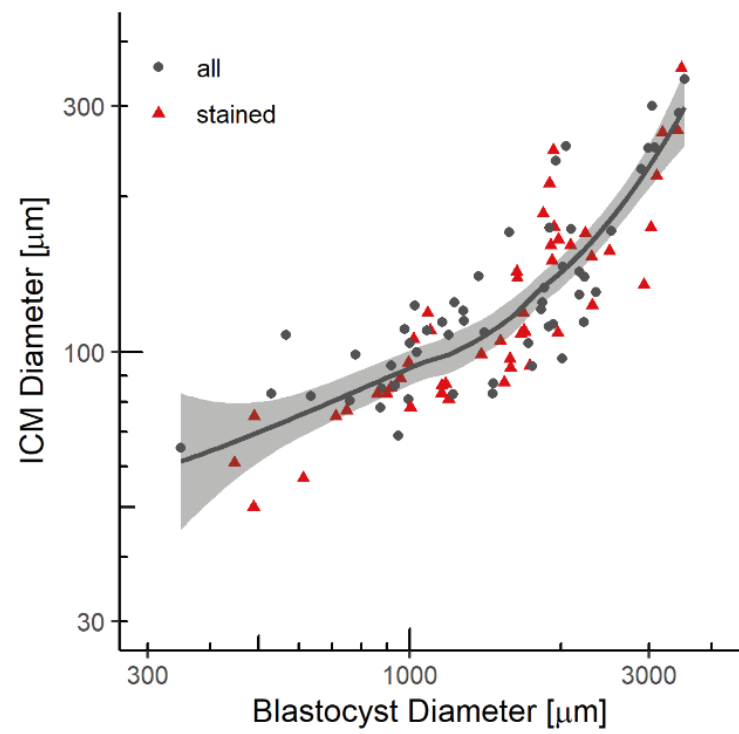

Figure 1: (A) Dark field images of six exemplary embryos showing the variability of embryo sizes during the sampling period. Images are shown at the same scale, the size indicates the respective diameter. (B) Embryo diameter and (C) ICM diameter of collected blastocysts in the course of diapause. In B and C, embryos selected for staining are shown with a red stroke. The y-axes are log-scaled. (D - E) Histogram showing the distribution of embryo diameters (D) and sampling dates (E) in all embryos and embryos selected for staining. (F) ICM diameter against embryo diameter. Note that $x$ and $y$ axis are log-scaled. The line represents the LOESS smooth of the transformed data with $95 \% \mathrm{Cl}$ as semi-transparent ribbon. 


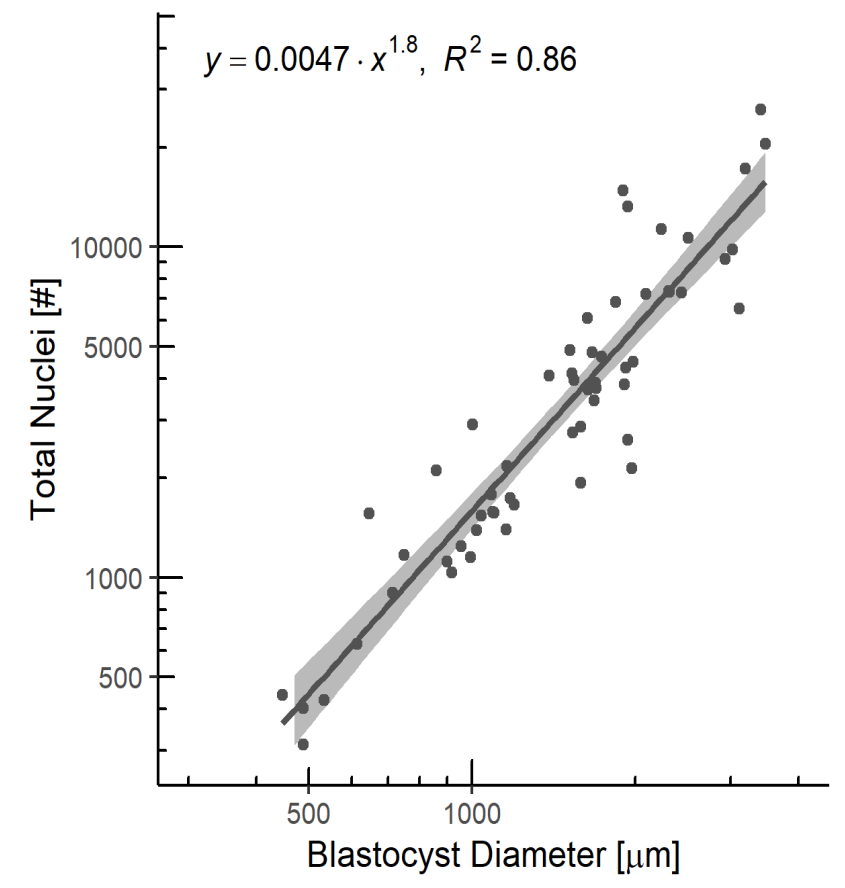

Figure 2: Correlation of total number of nuclei with embryo diameter, both plotted on a logarithmic scale. The line represents the linear fit of the transformed data with $95 \% \mathrm{Cl}$ as semi-transparent ribbon. The coefficients of the regression line equation have been backtransformed to the linear scale for easier interpretation.

A linear regression of the logarithm of the number of nuclei against time would estimate a doubling time of 18-21 days for TE cells and 21-26 days for the ICM cells. A doubling time of approximately 3 weeks illustrates well why mitotic cells were so far only rarely, if at all, observed in roe deer blastocysts during diapause $[4,11,12]$. However, these numbers include a certain error because even though the sampling date approximately reflects embryonic age, it does not per se reflect embryonic age. Fertilization in the roe deer is known to take place between mid-July and midAugust [27]. Therefore, the developmental age of each individual blastocyst varies considerably with respect to the sampling date. This variability and the lack of the known underlying distribution add further noise to any estimated growth model.

In contrast to the observations made using BrdU [12] and as described in previous histological studies [4, 11], we clearly observed mitotic cells in the ICM (Figure 3C). Since the number of mitotic cells observed was limited to individual blastocysts, we were not able to estimate a mitotic index. Depending on the exact stage and the compartment, mitotic indexes between $2 \%$ and $18 \%$ and estimated doubling times of 6-24h have been reported for developing mouse embryos [28-30]. Almost no dividing cells were observed in diapausing mouse blastocysts and the cell number remained stable [31, 32]. Thus, while our data clearly provides evidence for cell proliferation in both embryonic compartments, the number of cells dividing is largely reduced compared to nondiapausing murine blastocysts.

We observed that Ki67 stained nuclei were randomly distributed across both TE and ICM (Figure 4A). In the course of diapause, the fraction of Ki67 positive nuclei across all nuclei ranged from 0 to $30.6 \%$ with a mean of $7.2 \pm 0.7 \%$ Ki67 positive nuclei. With respect to the total number of nuclei, we observed a significant but only weak linear correlation for the fraction of Ki67 positive nuclei in both TE and ICM (Pearson's $\mathrm{R}=0.44, \mathrm{t}=3.59, \mathrm{df}=55, \mathrm{p}$-value $<0.001$ and Pearson's $\mathrm{R}=-0.33, \mathrm{t}=\mathrm{-}$ $2.58, \mathrm{df}=55, \mathrm{p}$-value $<0.05$, respectively, Figure $4 \mathrm{~B})$. Accordingly, the fraction of Ki67 positive nuclei showed a significant correlation with the sampling date (TE: Pearson's $R=0.26, t=2.01, d f=$ $55, p$-value $=0.05$, and ICM: Pearson's $R=-0.40, t=-3.26, d f=55, p$-value $<0.01$ ) (Figure 4C). Surprisingly, the average percentage of Ki67 positive cells in the ICM was significantly higher than in the TE $(16.6 \pm 1.5 \%$ versus $6.6 \pm 0.7 \%$, Mann-Whitney's U: $676.5, p<0.0001)$. This difference was more prominent in smaller than in larger blastocysts as seen in the LOESS fit (Figure 4). 
A

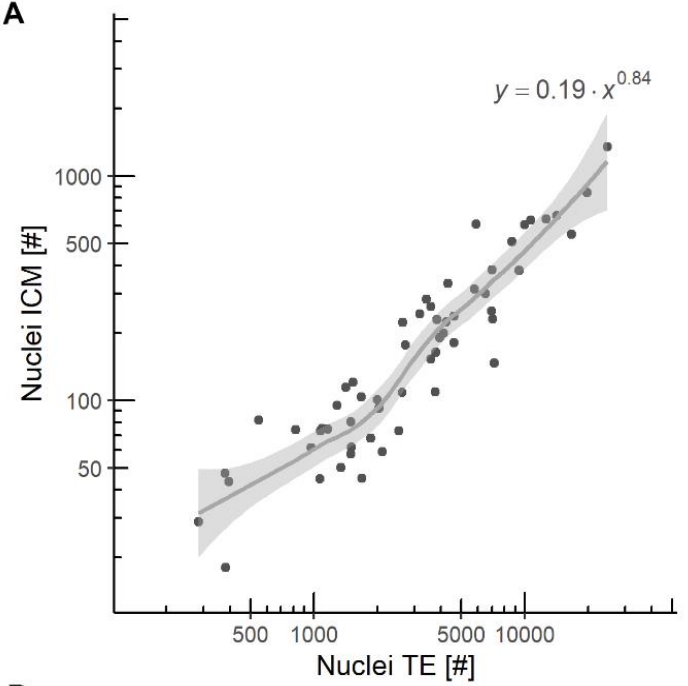

B

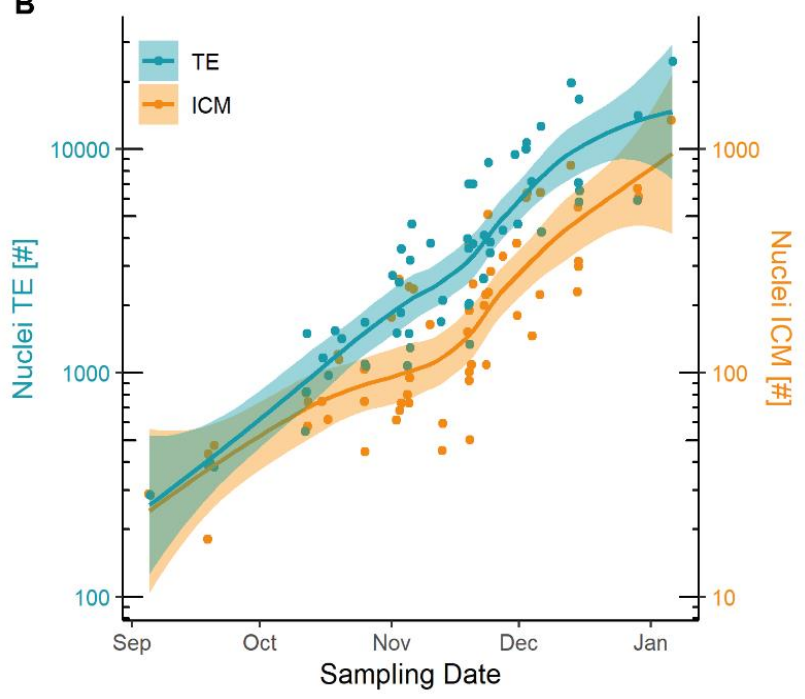

C

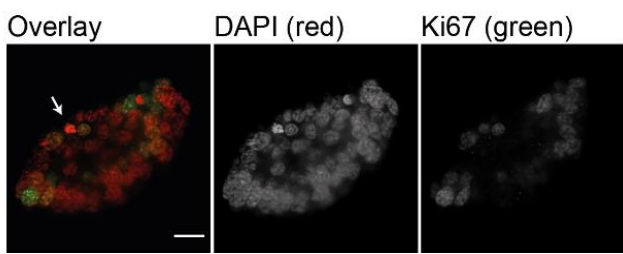

Figure 3: (A) Number of ICM nuclei against number of TE nuclei, both plotted on a logarithmic scale. The solid line represents the LOESS fit of the transformed data with $95 \% \mathrm{Cl}$ as semi-transparent ribbon. The coefficients of the regression line equation have been backtransformed to the linear scale for easier interpretation. (B) Number of TE nuclei (left axis, blue) or ICM nuclei (right axis, orange) against the sampling time. Left and right y-axis are on a logarithmic scale, with the left $y$-axis being shifted by one order of magnitude. The line represents the loess fit of the transformed data with $95 \% \mathrm{Cl}$ as semi-transparent ribbon. (C) Single, deconvolved optical section of an ICM with a mitotic cell (arrow). The scale bar represents $20 \mu \mathrm{m}$.

Interpreting Ki67 as a marker for proliferating cells, the results indicate a higher proliferation rate in the ICM than in the TE. Based on the rate of increase in the number of ICM versus TE nuclei (Figure 3B), one would expect the fraction Ki67 positive nuclei to be higher in the TE. At first sight, the two observations might thus seem contradictory. However, a recent study found that the level of Ki67 expression not only depends on the cell cycle stage, but also on the time previously spent in quiescence [15]. Thus, the duration of the G1 phase could potentially affect the expression of Ki67. A similar phenomenon has recently been described in diapausing mouse blastocysts harboring a FUCCl-reporter system. It was observed that the cells of the trophoblast arrested in the G1 phase of the cell cycle prior to the cells of the ICM. At reactivation, cells of the ICM re-entered the cell cycle prior to the trophoblast cells [14].

Additionally, the impact of Ki67 on cell cycle progression has been shown to vary between different cell types [14]. The analysed blastocysts consist of at least two (ICM and TE) or - depending on the 
stage of embryonic development - even more distinct cell types. Furthermore, it has been established that the differentiation of embryonic stem cells is linked to an increase in the duration of the G1 phase [33-35]. As the cells in the TE are likely further differentiated than cells of the ICM, they might display a longer $\mathrm{G} 1$ phase. We thus hypothesize that the overall duration of the cell cycle was longer in the ICM than in TE. Alternatively, the duration of the cell cycle was similar in both ICM and TE, but the fractional distribution of cell cycle phases differed, with TE cells having a longer $\mathrm{G} 1$ phase than ICM cells.

We noticed clear morphological differences between different ICM and accordingly assigned the blastocysts to four phenotypic groups, namely blastocysts with a round, spherical ICM $(n=5)$, an ellipsoid, often unilaterally flattened ICM $(n=21)$, an ICM with an internal cyst or a pit-like structure $(n=21)$ and a disk-shaped ICM $(n=10)$, respectively. Single $z$-slices for representative ICM of each group and an overview of the Imaris surface objects are displayed in Figure 5A and B, respectively. While there was no clear difference regarding the percentage of Ki67 positive nuclei within either the ICM or the TE (Figure 5F-G), the morphological groups differed with respect to the sampling date and the number of TE and ICM nuclei (Figure $5 \mathrm{C}-\mathrm{E}$ ). Based on our own and previous observations made by Keibel [11], we suggest that the morphological categories represent progressive developmental stages with a round ICM being the earliest and a disk ICM being the latest developmental stage observed.

The morphological changes occurring in the roe deer ICM are similar to those observed in bovine blastocysts collected on days 10-13 post fertilization [36, 37]. During this period, the Rauber's layer, which comprises TE cells that initially cover the epiblast, starts to dissolve and the spherical ICM develops into a flat embryonic disk [36-41]. At this point, the mesoderm induction commences and the primitive streak formation is initiated. The formation of an exteriorized embryonic disc is commonly observed among artiodactyls [42]. Yet, in most other artiodactyls the ICM simply flattens out. In mice, a similar pit-shape can be observed before the egg-cylinder stage is formed [43]. Whether these two stages could be functionally comparable remains to be determined.

We noted a tendency of the fraction of Ki67 positive cells to decrease in the ICM with further developmental progression, with a significant decrease between cyst and disk shaped ICM (Figure 5 D). Comparable patterns for the number of proliferating cells have been observed in the ectoderm, endoderm, and mesoderm of developing mouse embryos [28-30]. Additionally, differentiation of murine embryonic stem cells also leads to an increase in the duration of the G1 phase [33-35]. It is thus possible that cell differentiation might be the reason for the reduction in the fraction of Ki67 positive cells.

Taken together, the morphological changes in the ICM might also be indicative of ongoing differentiation in the ICM during diapause. Further verification of differentiation factors is needed to verify this hypothesis. 
A Overlay

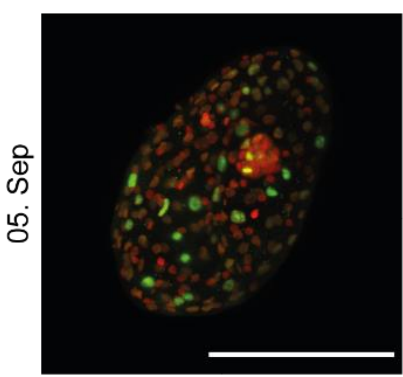

DAPI (red)
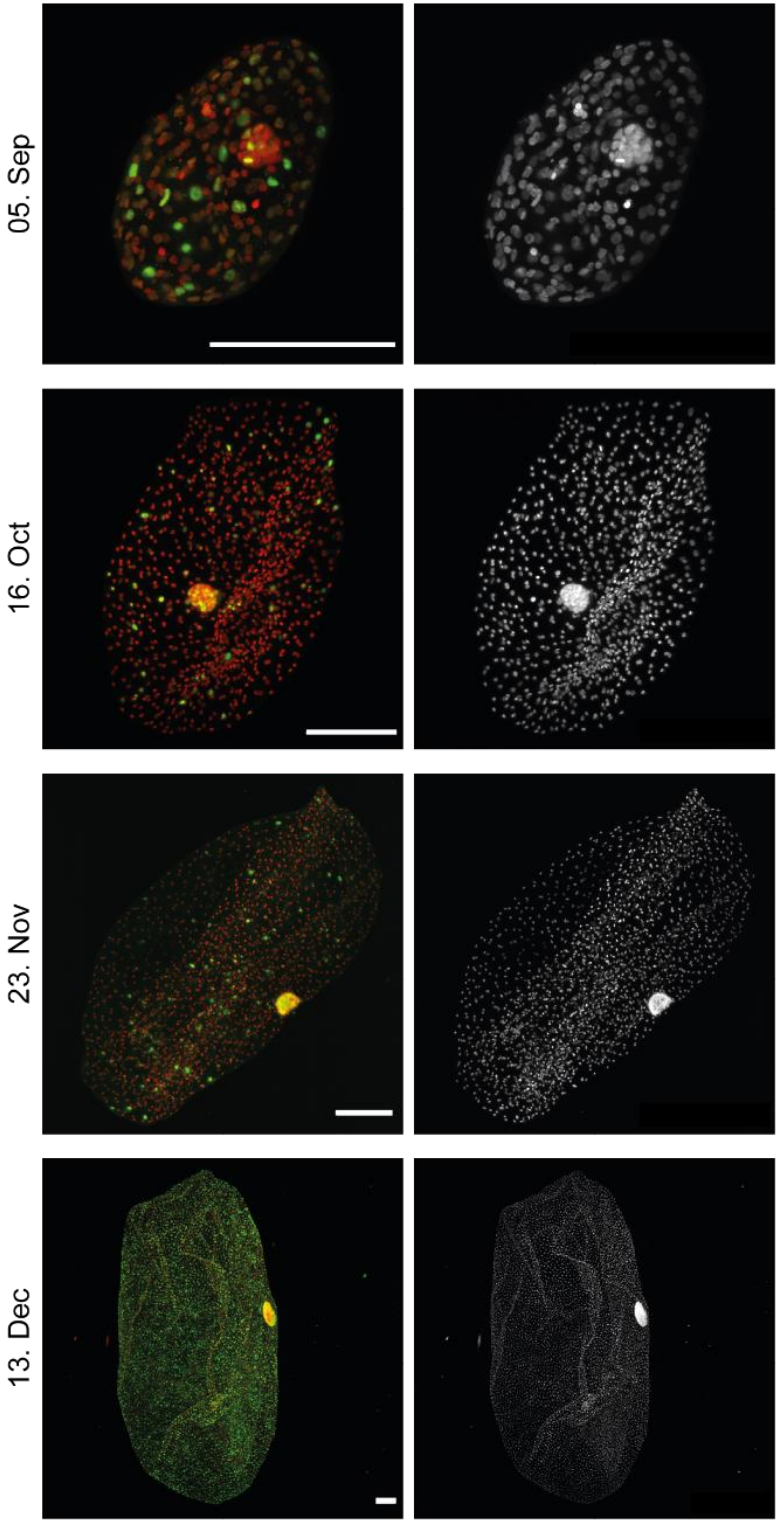

B

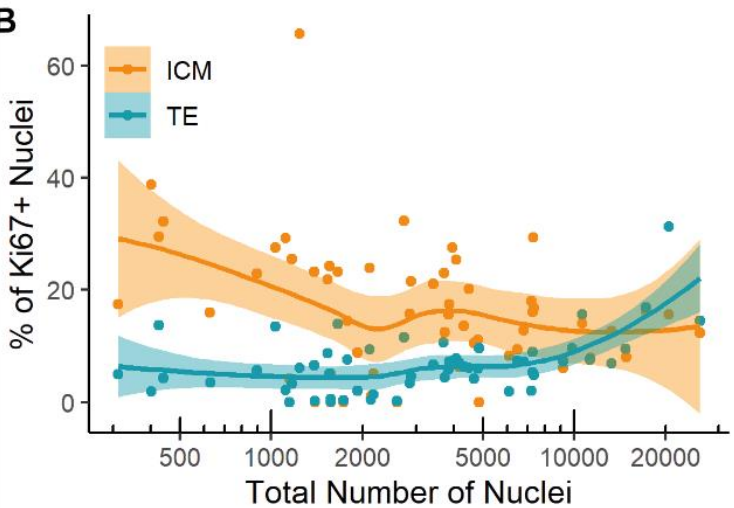

C

0

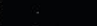

Ki67 (green)
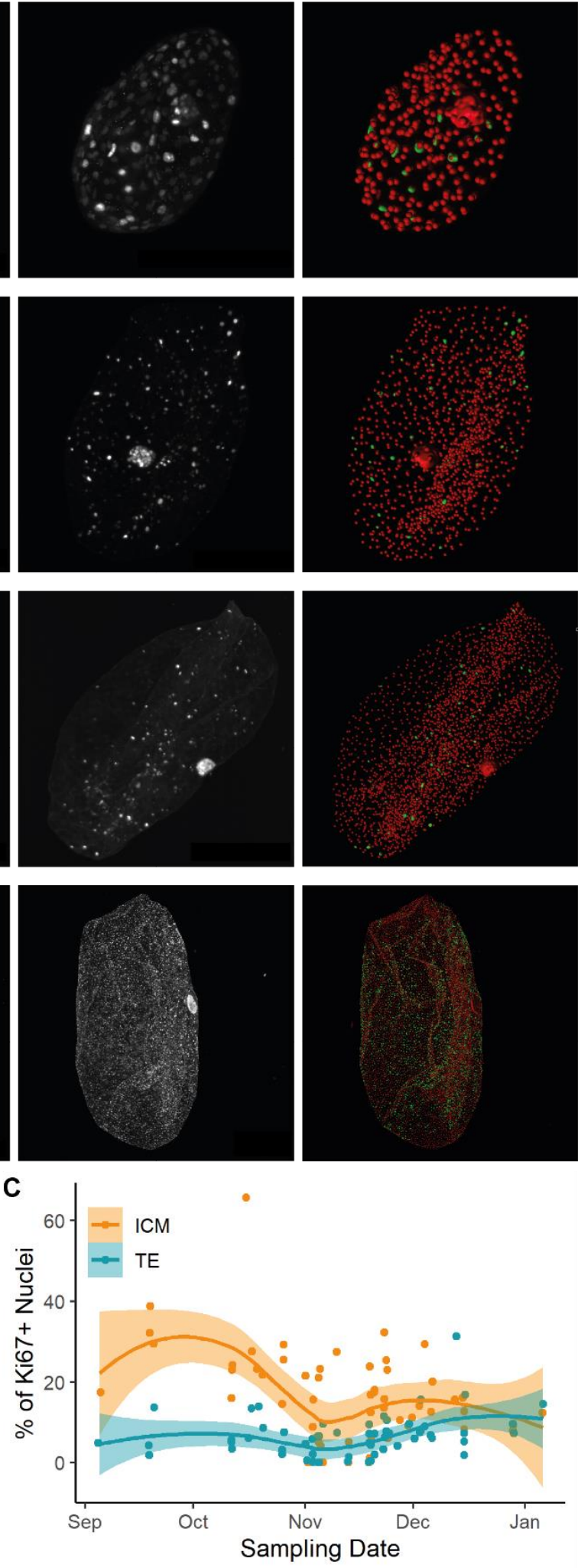

Segmentation
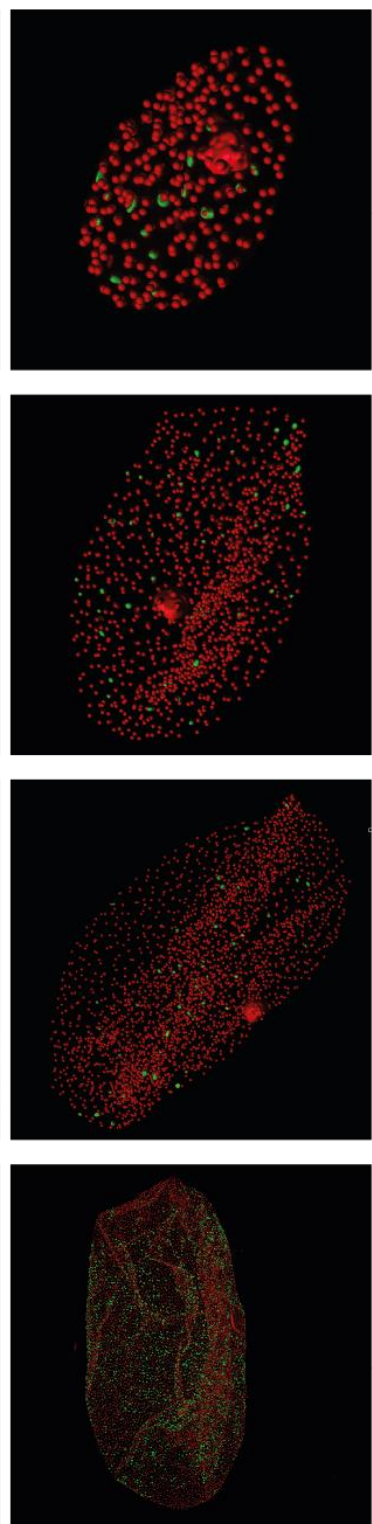

Sampling Date

Figure 4: (A) Maximum Z projection of light sheet images from four exemplary embryos analysed (overlay, DAPI, Ki67) and visualization of segmentation with Imaris (right). The scale bar represents $200 \mu \mathrm{m}$. (B - C) Fraction of Ki67 positive nuclei in either ICM (orange) or TE (blue) with respect to (B) total number of nuclei and (C) sampling date. The line in (B) and (C) represents the loess smooth with $95 \% \mathrm{Cl}$ as semi-transparent ribbon. 
A
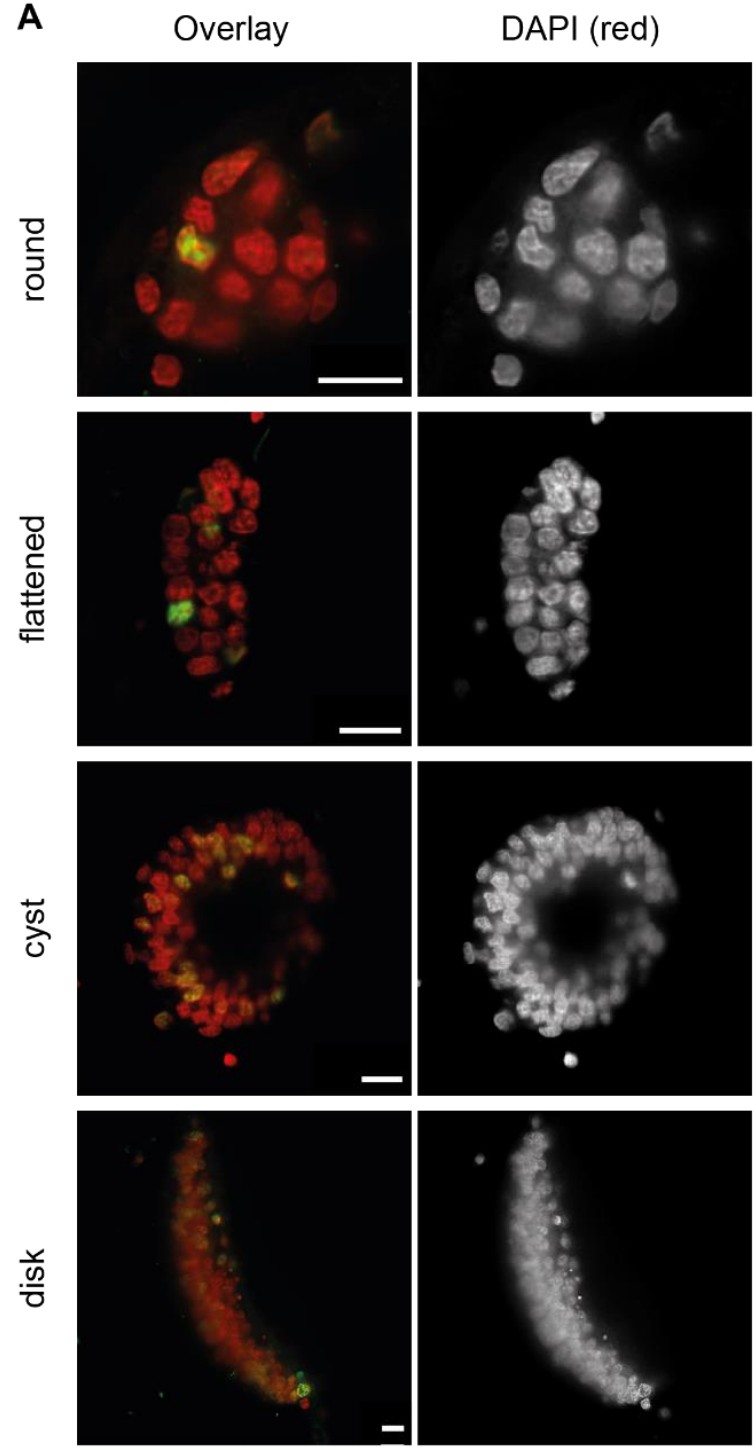

B

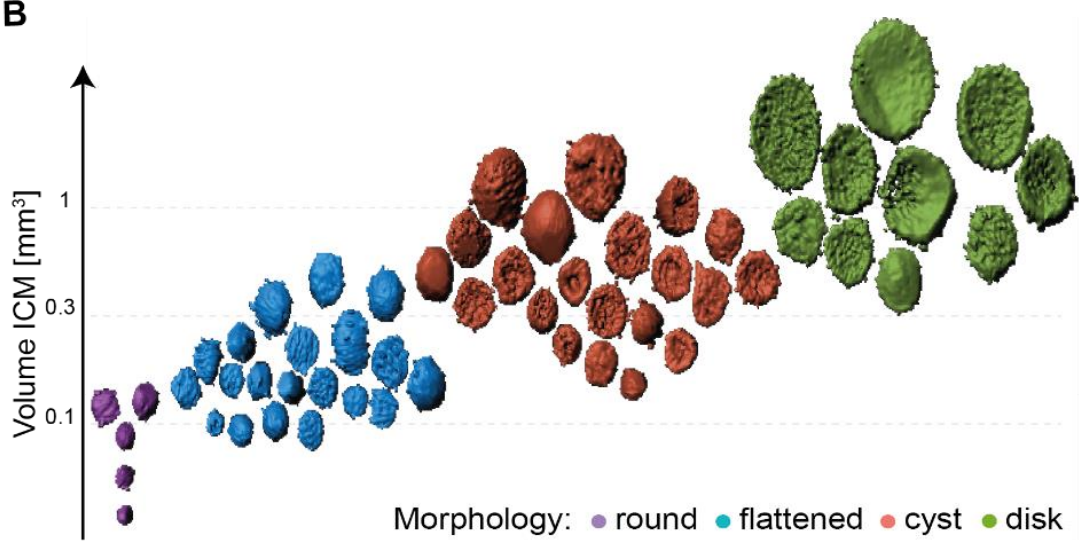

C

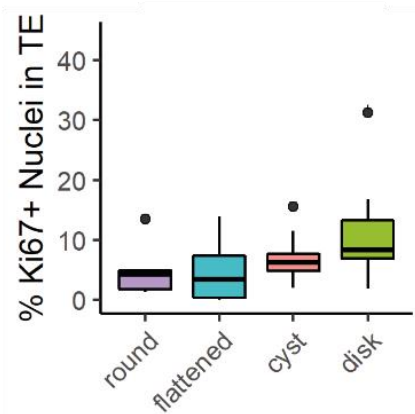

D
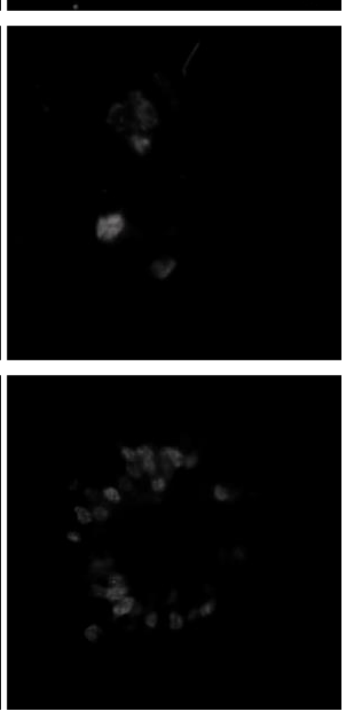

E

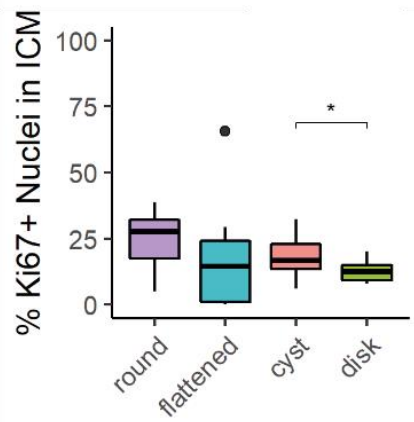

$$
\text { है }
$$

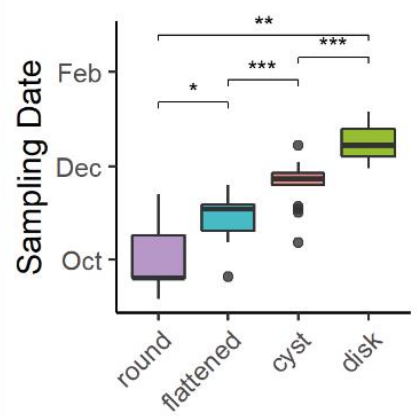

$\mathbf{F}$
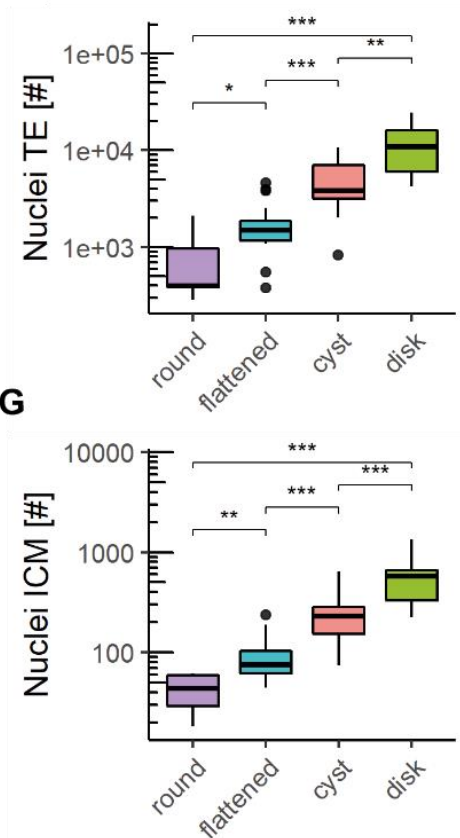

Figure 5: (A) Deconvolved lightsheet images for different ICM morphologies, scale bar represents $20 \mu \mathrm{m}$. (B) Visualization of ICM surface segmentation in Imaris, color code represents the morphological characterization. (C-G) Boxplots for sampling date (C), number of nuclei in TE (D) and ICM (E), and percentage of Ki67 positive nuclei in TE (F) and ICM (G). Significance levels of Mann-Whitney-U-Test: $n s: p>$ $0.05, *: p<=0.05, * *: p<=0.01, * * *: p<=0.001$. 


\section{Conclusions}

Our results demonstrate a continuous increase in the number of nuclei in both TE and ICM in the course of diapause in roe deer. Although the relative increase of nuclei in the ICM was lower than in the TE, we provide evidence of a higher proportion of Ki67 positive cells in the ICM than in the TE. This may be either due to a different basal expression of Ki67 in the two embryonic compartments or due to a different distribution of cell cycle phases among cells of ICM versus TE. Additional cell cycle markers are needed to distinguish whether the fractional distribution of cell cycle phases differs in cells of the ICM and TE.

In addition to the differences in proliferation between TE and ICM, we confirmed that the roe deer ICM undergoes clear morphological changes during diapause. Markers for endoderm differentiation (e.g. GATA4, GATA6, SOX17) and mesendoderm (GSC, NODAL, MIXL1) and mesoderm induction (e.g. BRY/T, EOMES) are needed to gain deeper insights into ongoing developmental processes.

In this study, we focused on the period of pre-elongation and did not include elongated blastocysts. The extreme difference in tissue thickness and size between blastocysts and elongated blastocysts would have required different staining and imaging techniques leading to difficulties in adequately comparing the findings. However, we assume from the increase in blastocyst size $[44,45]$ that additional and most likely more pronounced differences would have been evident between diapausing and elongating blastocysts. It would be very informative to determine the point of transition as it most likely indicates embryo reactivation.

Studying the decelerating and arresting development as well as cell cycle progression will contribute to the improvement of existing artificial reproduction technology and shed light on cellular homeostasis in physiological and pathological contexts. Future studies focusing on the molecular mechanism of slow developmental progression are thus essential.

\section{Declaration of conflict of interest}

The authors declare that there is no conflict of interest that could be perceived as prejudicing the impartiality of this article.

\section{Funding}

This study was funded by the Swiss National Science Foundation SNSF (31003A_159734 and 310030_185026).

\section{Acknowledgements}

We would like to express our gratitude to our group members and in particular the local hunters, who participated during the collection of samples for this study. We specifically thank Martina Helbling and David Kopps for assistance in sample processing. In addition, we thank Szymon Stoma for his valuable input regarding the image analysis and acknowledge the assistance and support of the Center for Microscopy and Image Analysis, University of Zurich. The authors are active participants of the COST Action CA16119 (CellFit - In vitro 3D total cell guidance and fitness). 


\section{References}

[1] Renfree MB and Fenelon JC. 2017 The enigma of embryonic diapause. Development 144(18) 3199210.

[2] Bischoff TLW. 1854 Entwicklungsgeschichte des Rehes: Walter De Gruyter Incorporated.

[3] Flint AP, Krzywinski A, Sempéré AJ, Mauget R and Lacroix A. 1994 Luteal oxytocin and monoestry in the roe deer Capreolus capreolus. Journal of reproduction and fertility 101 651-6.

[4] Short RV and Hay MF. 1966 Delayed implantation in the roe deer (Capreolus capreolus). Symp Zool Soc Lond.15 173-92.

[5] Aitken RJ. 1981 Aspects of delayed implantation in the roe deer (Capreolus capreolus). J Reprod Fertil Suppl 29 83-95.

[6] Sempéré AJ, Mauget R and Chemineau P. 1992 Experimental induction of luteal cyclicity in roe deer (Capreolus capreolus). Journal of reproduction and fertility 96 379-84.

[7] Hoffmann B, Barth D and Karg H. 1978 Progesterone and estrogen levels in peripheral plasma of the pregnant and nonpregnant roe deer (Capreolus capreolus). Biology of reproduction 19 931-5.

[8] Degrelle SA, Campion E, Cabau C, Piumi F, Reinaud P, Richard C, et al. 2005 Molecular evidence for a critical period in mural trophoblast development in bovine blastocysts. Dev Biol 288(2) 448-60.

[9] Ulbrich SE, Schulke K, Groebner AE, Reichenbach HD, Angioni C, Geisslinger G, et al. 2009 Quantitative characterization of prostaglandins in the uterus of early pregnant cattle. Reproduction 138371 82.

[10] Brooks K, Burns G and Spencer TE. 2014 Conceptus elongation in ruminants: roles of progesterone, prostaglandin, interferon tau and cortisol. J Anim Sci Biotechnol 5(1) 53.

[11] Keibel F. 1902 Die Entwicklung des Rehes bis zur Anlage des Mesoblast. Archiv für Anatomie und Physiologie Anatomische Abteilung. (1902) 292-314.

[12] Lengwinat T and Meyer HH. 1996 Investigations of BrdU incorporation in roe deer blastocysts in vitro. Anim Reprod Sci 45(1-2) 103-7.

[13] Scholzen T and Gerdes J. 2000 The Ki-67 protein: from the known and the unknown. J Cell Physiol 182(3) 311-22.

[14] Sun X and Kaufman PD. 2018 Ki-67: more than a proliferation marker. Chromosoma 127(2) 175-86.

[15] Miller I, Min M, Yang C, Tian C, Gookin S, Carter D, et al. 2018 Ki67 is a Graded Rather than a Binary Marker of Proliferation versus Quiescence. Cell Rep 24(5) 1105-12 e5.

[16] Beyes M, Nause N, Bleyer M, Kaup F-J and Neumann S. 2017 Immunohistochemical Investigation of the Proliferation Activity in European Roe Deer (Capreolus capreolus) during Embryogenesis after Diapause by Using Ki-67. Journal of Zoological Sciences 5(2) 68-76.

[17] Blottner S and Schoen J. 2005 Minimal activity in both proliferation and apoptosis of interstitial cells indicates seasonally persisting Leydig cell population in roe deer. Cell Tissue Res 321(3) 473-8.

[18] Wolfs TG, Kramer BW, Thuijls G, Kemp MW, Saito M, Willems MG, et al. 2014 Chorioamnionitisinduced fetal gut injury is mediated by direct gut exposure of inflammatory mediators or by lung inflammation. Am J Physiol Gastrointest Liver Physiol 306(5) G382-93.

[19] Kuypers E, Collins JJ, Jellema RK, Wolfs TG, Kemp MW, Nitsos I, et al. 2012 Ovine fetal thymus response to lipopolysaccharide-induced chorioamnionitis and antenatal corticosteroids. PLoS One 7(5) e38257.

[20] Walton RM, Parmentier T and Wolfe JH. 2013 Postnatal neural precursor cell regions in the rostral subventricular zone, hippocampal subgranular zone and cerebellum of the dog (Canis lupus familiaris). Histochem Cell Biol 139(3) 415-29.

[21] Salvadori C, Svara T, Rocchigiani G, Millanta F, Pavlin D, Cemazar M, et al. 2017 Effects of Electrochemotherapy with Cisplatin and Peritumoral IL-12 Gene Electrotransfer on Canine Mast Cell Tumors: a Histopathologic and Immunohistochemical Study. Radiol Oncol 51(3) 286-94.

[22] Vermeulen M, Del Vento F, de Michele F, Poels J and Wyns C. 2018 Development of a Cytocompatible Scaffold from Pig Immature Testicular Tissue Allowing Human Sertoli Cell Attachment, 
Proliferation and Functionality. Int J Mol Sci 19(1).

[23] Schindelin J, Arganda-Carreras I, Frise E, Kaynig V, Longair M, Pietzsch T, et al. 2012 Fiji: an opensource platform for biological-image analysis. Nat Methods 9(7) 676-82.

[24] R Core Team. 2017 R: A language and environment for statistical computing. R Foundation for Statistical Computing.

[25] Wickham H. 2016 ggplot2: elegant graphics for data analysis: Springer.

[26] Aitken RJ, Burton J, Hawkins J, Kerr-Wilson R, Short RV and Steven DH. 1973 Histological and Ultrastructural Changes in the Blastocyst and Reproductive Tract of the Roe Deer, Capreolus Capreolus, during Delayed Implantation. Reproduction 34(3) 481-93.

[27] Aitken RJ. 1974 Delayed implantation in roe deer (Capreolus capreolus). J Reprod Fertil 39(1) 22533.

[28] Solter D, Škreb N and Damjanov I. 1971 Cell cycle analysis in the mouse egg-cylinder. Experimental Cell Research 64(2) 331-4.

[29] Snow MHL. 1976 Embryo Growth During the Immediate Postimplantation Period. In: Katherine E, Maeve OC, editors. Embryogenesis in Mammals Ciba Foundation. p. 53-70.

[30] Handyside AH and Hunter S. 1986 Cell division and death in the mouse blastocyst before implantation. Roux's archives of developmental biology 195(8) 519-26.

[31] Kamemizu C and Fujimori T. 2019 Distinct dormancy progression depending on embryonic regions during mouse embryonic diapausedagger. Biol Reprod 100(5) 1204-14.

[32] Copp AJ. 1982 Effect of implantational delay on cellular proliferation in the mouse blastocyst. J Reprod Fertil 66(2) 681-5.

[33] Waisman A, Sevlever F, Elias Costa M, Cosentino MS, Miriuka SG, Ventura AC, et al. 2019 Cell cycle dynamics of mouse embryonic stem cells in the ground state and during transition to formative pluripotency. Sci Rep 9(1) 8051.

[34] Roccio M, Schmitter D, Knobloch M, Okawa Y, Sage D and Lutolf MP. 2013 Predicting stem cell fate changes by differential cell cycle progression patterns. Development 140(2) 459-70.

[35] Coronado D, Godet M, Bourillot PY, Tapponnier Y, Bernat A, Petit M, et al. 2013 A short G1 phase is an intrinsic determinant of naive embryonic stem cell pluripotency. Stem Cell Res 10(1) 118-31.

[36] van Leeuwen J, Berg DK and Pfeffer PL. 2015 Morphological and Gene Expression Changes in Cattle Embryos from Hatched Blastocyst to Early Gastrulation Stages after Transfer of In Vitro Produced Embryos. PLoS One 10(6) e0129787.

[37] Maddox-Hyttel P. 2003 Immunohistochemical and ultrastructural characterization of the initial posthatching development of bovine embryos. Reproduction 125(4) 607-23.

[38] Tveden-Nyborg P, Peura TT, Hartwich KM, Walker SK and Maddox-Hyttel P. 2005 Morphological characterization of pre- and peri-implantation in vitro cultured, somatic cell nuclear transfer and in vivo derived ovine embryos. Reproduction 130(5) 681-94.

[39] Guillomot M, Turbe A, Hue I and Renard JP. 2004 Staging of ovine embryos and expression of the Tbox genes Brachyury and Eomesodermin around gastrulation. Reproduction 127(4) 491-501.

[40] Viebahn C, Stortz C, Mitchell SA and Blum M. 2002 Low proliferative and high migratory activity in the area of Brachyury expressing mesoderm progenitor cells in the gastrulating rabbit embryo. Development 129(10) 2355-65.

[41] Flechon JE, Degrouard J and Flechon B. 2004 Gastrulation events in the prestreak pig embryo: ultrastructure and cell markers. Genesis 38(1) 13-25.

[42] Stern CD. 2004 Gastrulation: From Cells to Embryo. N. Y.: Cold Spring Harbor Laboratory Press.

[43] Harrison SE, Sozen B, Christodoulou N, Kyprianou C and Zernicka-Goetz M. 2017 Assembly of embryonic and extraembryonic stem cells to mimic embryogenesis in vitro. Science 356(6334).

[44] Drews B, Rudolf Vegas A, van der Weijden VA, Milojevic V, Hankele AK, Schuler G, et al. 2019 Do ovarian steroid hormones control the resumption of embryonic growth following the period of diapause in roe deer (Capreolus capreolus)? Reprod Biol 19(2) 149-57. 
[45] van der Weijden VA and Ulbrich SE. Embryonic diapause in the European roe deer (Capreolus capreolus). submitted.

\section{Audience Discussion}

Unknown questioner: I just have a question, because you just started with 6 embryos. Will you continue the study? You chose probably chose embryos at different stages of diapause, so did you notice some trend of decrease or increase of proliferation in time or is it too early to say?

Anna Ruegg: So, from the impressions that I have from the images, I couldn't say whether there was a clear trend. I think I really need to perform the quantification, because we do see more red spots, but there are much more cells. We really need to quantify how much this is in numbers and the percentage of cells which are positive. This will reflect better what is going on.

Grazyna Ptak: Is the development of the roe deer really slow and did you try to compare it to other deer, which are not diapausing, like fallow deer or red deer? Because maybe cow embryos are not the perfect control in this case?

Susanne Ulbrich: You're right, not much is known about other species. The fallow deer would be the most likely one to be easily studied, we like this idea as well. We think that the relatively late implantation in cows would also comprise the term "slow", but yet nobody says the embryo of the cow has a diapause, simply - it implants only 18 days after fertilization. So, "slow", and I would put it in parenthesis, what is slow? Yes, slower than other species which are well characterized, but, good point, it might not be different from other species.

Jane Fenelon: Do you know when embryonic genome activation occurs in the roe deer?

Susanne Ulbrich: Our data is very scarce regarding the very early development in vivo. From all the hunting we have no idea how old the embryos are. So, we don't know when fertilization exactly takes place. This is partly why we think our in vitro culture/production is so important, because that would be one of the questions we want to answer.

Jane Fenelon: Do you think the slow delay of the roe deer in culture is partly due to the culture medium, because the embryo didn't look overly happy.

Susanne Ulbrich: True, yes, we don't know. The only indication we have in assuming that this might be not due to the in vitro culture are the in vivo findings. But again, I share possible concerns regarding the superovulation protocols. So, we don't know. We hope if we molecularly characterize the developing embryos in vitro, whether or not they are parthenogenetic and whether pace they take regarding their gene expression is alike other species. This is partly what Sandra will do in the future.

Jane Fenelon: Do you think there are distinct stages in the diapause in term of early, mid and late diapause, or is it really just a slowing down of the development as opposed to this is diapause?

Susanne Ulbrich: In the very early phase of diapause, we can't get samples from hunting, because the hunting season isn't open and we simply don't know whether they have bred or not. For me it's very interesting to see in vitro culture results. I thought that the embryo, upon entering into the uterus, hatching from the zona, is inhibited to grow and thereby this would determine the beginning of diapause. We were very excited and surprised to see that the embryo upon enters the uterus in a fairly earlier stage than we had expected. That implies that it might be slow in the development from the beginning on, but yet not so slow as you would expect during diapause. During diapause, we hope to distinguish different phases of development, Vera will show this. 
Unknown questioner: There are all these distinguished phases during the embryonic diapause and those phases are based on the morphology. You collected the uterine fluid at the same time, right and your staging was based on the morphology?

Susanne Ulbrich: Initially, the only thing we could do is characterize the morphology regarding the size. Later, we extracted the DNA and considered the cell count. But distinguishing the cell count of inner cell mass and the trophectoderm is thereby difficult. The question whether we have distinct phases or whether we consider a continuous development requires either a technical or a conceptual approach. We do this in trying to mirror both sides, the embryonic side, the uterine side and the histotrop (I like to emphasize the difference between histotroph and histotrop). The uterine flushing as a substrate has trophic and trophic effects. I think that we hopefully can deduce the factors that induce morphological changes. To come to your point; we're looking for something, but we don't know yet what it is.

Katarina Jewgenow: Other than P4 and PR which you reported upon, did you think about using antigestagens to block progesterone at its receptor?

Susanne Ulbrich: There are earlier studies which followed individual roe deers across the season and this is something we want to do. Other than the gestagen protesteron, we are very interested in and Anna Hankele is working on neurogestagens, so 5-alpha and 3-alpha-reduced and 20-alpha-reduced metabolites of gestagens. These could also take part in this concerted action of whatever the $\mathrm{CL}$ is synthesizing. Thus, about antigestagen and the role of P4, despite others, currently we will start to first characterize the spectrum of gestagens. Any experimental approach is more difficult, but we also hope to apply the antigestagens or even to induce luteolysis to see whether this obligatory phase of diapause is really needed or whether we can transfer an embryo later and then have a shortened diapause period. 